\title{
RESEARCH
}

Open Access

\section{Genetic associations of in vivo pathology influence Alzheimer's disease susceptibility}

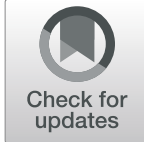

Jieun Seo ${ }^{1 \dagger}$, Min Soo Byun ${ }^{2 \dagger}$, Dahyun $\mathrm{Yi}^{3}$, Jun Ho Lee ${ }^{4}$, So Yeon Jeon ${ }^{5}$, Seong A. Shin ${ }^{6}$, Yu Kyeong Kim ${ }^{6}$, Koung Mi Kang ${ }^{7}$, Chul-Ho Sohn ${ }^{7}$, Gijung Jung ${ }^{8}$, Jong-Chan Park ${ }^{1,9}$, Sun-Ho Han ${ }^{1,9}$, Jayoung Byun ${ }^{10}$, Inhee Mook-Jung ${ }^{1,9}$, Dong Young Lee ${ }^{3,7,11^{*}}$, Murim Choi ${ }^{1^{*}}$ (D) and for the KBASE Research Group

\begin{abstract}
Introduction: Although the heritability of sporadic Alzheimer's disease (AD) is estimated to be $60-80 \%$, addressing the genetic contribution to $A D$ risk still remains elusive. More specifically, it remains unclear whether genetic variants are able to affect neurodegenerative brain features that can be addressed by in vivo imaging techniques.

Methods: Targeted sequencing analysis of the coding and UTR regions of 132 AD susceptibility genes was performed. Neuroimaging data using ${ }^{11} \mathrm{C}$-Pittsburgh Compound B positron emission tomography (PET), ${ }^{18} \mathrm{~F}-$ fluorodeoxyglucose PET, and MRI that are available from the KBASE (Korean Brain Aging Study for Early Diagnosis and Prediction of Alzheimer's disease) cohort were acquired. A total of 557 participants consisted of 336 cognitively normal (CN) adults, 137 mild cognitive impairment (MCI), and 84 AD dementia (ADD) groups.

Results: We called 5391 high-quality single nucleotide variants (SNVs) on AD susceptibility genes and selected significant associations between variants and five in vivo AD pathologies: (1) amyloid $\beta$ (Aß) deposition, (2) ADsignature region cerebral glucose metabolism (AD-Cm), (3) posterior cingulate cortex (PCC) cerebral glucose metabolism (PCC-Cm), (4) AD-signature region cortical thickness (AD-Ct), and (5) hippocampal volume (Hv). The association analysis for common variants (allele frequency (AF) > 0.05) yielded several novel loci associated with $A \beta$ deposition (PIWIL1-rs10848087), AD-Cm (NME8-rs2722372 and PSEN2-rs75733498), AD-Ct (PSEN1-rs7523) and, Hv (CASS4-rs3746625). Meanwhile, in a gene-based analysis for rare variants (AF < 0.05), cases carrying rare variants in LPL, FERMT2, NFAT5, DSG2, and ITPR1 displayed associations with the neuroimaging features. Exploratory voxel-based brain morphometry between the variant carriers and non-carriers was performed subsequently. Finally, we document a strong association of previously reported APOE variants with the in vivo AD pathologies and demonstrate that the variants exert a causal effect on $A D$ susceptibility via neuroimaging features.
\end{abstract}

Conclusions: This study provides novel associations of genetic factors to $A \beta$ accumulation and AD-related neurodegeneration to influence AD susceptibility.

Keywords: Alzheimer's disease, Targeted panel sequencing, Genetic association, Neuroimaging, In vivo AD pathologies, PET, MRI

\footnotetext{
* Correspondence: selfpsy@snu.ac.kr; murimchoi@snu.ac.kr

${ }^{\dagger}$ Jieun Seo and Min Soo Byun contributed equally to this work.

${ }^{3}$ Institute of Human Behavioral Medicine, Medical Research Center Seoul National University, Seoul, Republic of Korea

'Department of Biomedical Sciences, Seoul National University College of Medicine, Seoul, Republic of Korea

Full list of author information is available at the end of the article
}

\section{$\triangle B M C$}

(c) The Author(s). 2020 Open Access This article is licensed under a Creative Commons Attribution 4.0 International License, which permits use, sharing, adaptation, distribution and reproduction in any medium or format, as long as you give appropriate credit to the original author(s) and the source, provide a link to the Creative Commons licence, and indicate if changes were made. The images or other third party material in this article are included in the article's Creative Commons licence, unless indicated otherwise in a credit line to the material. If material is not included in the article's Creative Commons licence and your intended use is not permitted by statutory regulation or exceeds the permitted use, you will need to obtain permission directly from the copyright holder. To view a copy of this licence, visit http://creativecommons.org/licenses/by/4.0/. The Creative Commons Public Domain Dedication waiver (http://creativecommons.org/publicdomain/zero/1.0/) applies to the data made available in this article, unless otherwise stated in a credit line to the data. 


\section{Background}

Alzheimer's disease (AD), the most common cause of dementia, is a neurodegenerative disease with high heritability estimated to be $60-80 \%[1,2]$. Previous genetic studies have elucidated causative rare variants contributing to familial AD that commonly occurs before the age of 65 (early onset). These variants are found in genes related to amyloid beta (A $\beta$ ) synthesis, such as APP (amyloid precursor protein), PSEN1 (presenilin 1), and PSEN2 (presenilin 2) [3]. However, as early-onset familial AD accounts for $<5 \%$ of $\mathrm{AD}$ cases [2,3], unraveling the complex genetic contributions to sporadic AD cases, which represents the majority of $\mathrm{AD}$ cases that occur after age 65 (late onset), is important. To date, apolipoprotein $\mathrm{E}(A P O E) \varepsilon 4$ allele (APOE4) is the strongest genetic factor of sporadic AD [4], which confers a 3- to 15fold increased risk of $\mathrm{AD}$ [5]. Recent large-scale genome-wide association studies (GWAS) have identified novel common variants of AD in loci involved in amyloid synthesis, nervous system development, synaptic transmission, and inflammation pathways [4, 6-9]. Nevertheless, APOE4 and several other common variants identified by previous GWAS explain a modest fraction of AD heritability [4], and addressing the remainder still remains as a challenge.

Most of the previous GWAS relied on phenotypes defined by clinical diagnosis of AD dementia (ADD) [8] and not by AD pathology or its surrogate biomarkers. Therefore, it remains unclear whether previously reported genes exert associations through modulating in vivo AD pathologies. Also, applying next-generation sequencing (NGS) technologies increases the possibility of detecting rare variants with large effect on diseases, which may help to explain the missing heritability [2, 10]. Although recent efforts to aggressively integrate genome-wide effects of common and rare variants has substantially improved our understanding of $\mathrm{AD}$ genetics [11, 12], how individual variants function to confer such effects still requires further investigation. A number of previous studies investigated genetic association with in vivo $\mathrm{AD}$ pathologies using $\mathrm{AD}$ biomarkers, but none has utilized multiple in vivo $A D$ pathology features and integrated them with common and rare genetic variants [13-18].

Although genome-wide approaches enable unbiased screening of novel variants, we chose to perform a targeted sequencing (TS) approach as it provided the following advantages. First, overarching hypothesis of the study assumes that there are quantifiable contributions of imaging biomarkers on the known genetic signals for LOAD, thereby aiming to provide clues of variant functions. Therefore, we decided to focus our analyses on the selected 132 genes with previous associations with LOAD. Second, compared to genome-wide approaches, the number of data points in TS is substantially smaller and allows reduced statistical penalty. Lastly, TS reduces sequencing cost and allows easier data handling.

Based on an imaging genetics approach, this work aimed to identify common and rare genetic variants closely associated with cerebral $\mathrm{A} \beta$ deposition and $\mathrm{AD}$ type neurodegeneration by conducting a TS analysis of 132 AD-related genes in 557 deep-phenotyped participants with multi-modal brain imaging information including $\left[{ }^{11} \mathrm{C}\right]$ Pittsburgh Compound B (PiB)-positron emission tomography (PET), $\left[{ }^{18} \mathrm{~F}\right]$ fluorodeoxyglucose (FDG)-PET, and magnetic resonance imaging (MRI). Our multivariable analysis approach provides a more accurate model for AD susceptibility by comparing with biomarkers for in vivo AD pathology.

\section{Materials and methods Participants}

We evaluated 557 participants who were recruited by the Korean Brain Aging Study for the Early Diagnosis and Prediction of Alzheimer's disease (KBASE), a prospective cohort study initiated in 2014 (Supplementary Table S1) [13]. Participants consisted of 274 cognitively normal (CN) older adults (CN-old, age $\geq 55$ ), $62 \mathrm{CN}$ young and middle-aged adult $(\mathrm{CN}$-ym, age $<55), 137$ individuals with mild cognitive impairment (MCI), and 84 patients with ADD. Details on the recruitment and inclusion/exclusion criteria are described in the Supplementary Methods.

\section{Evaluation of in vivo $A D$ pathologies}

All participants underwent comprehensive clinical and neuropsychological evaluations and multi-modal brain imaging (including $\left[{ }^{11} \mathrm{C}\right] \mathrm{PiB}-\mathrm{PET},\left[{ }^{18} \mathrm{~F}\right] \mathrm{FDG}-\mathrm{PET}$, and MRI). Blood was sampled for DNA extraction. APOE genotyping was conducted as previously described [18]. More detailed information on the standardized assessment used in the KBASE cohort is described in a previous report [13]. For surrogate markers of in vivo cerebral $\mathrm{A} \beta$ deposition, we quantified the standardized uptake value ratio (SUVR) of global $\left[{ }^{11} \mathrm{C}\right] \mathrm{PiB}$ retention level in the cortical region-of-interests (ROIs). $\left[{ }^{18} \mathrm{~F}\right] \mathrm{FDG}$ PET was used to measure cerebral glucose metabolism in the $\mathrm{AD}$-signature regions $(\mathrm{AD}-\mathrm{Cm})$ and posterior cingulate cortex (PCC-cm), which can capture early regional metabolic and functional deficits related to $\mathrm{AD}$ processes $[14,15]$. T1 MRI was used to evaluate cortical thickness in the AD-signature regions (AD-Ct) [19] and intracranial volume-adjusted hippocampal volume (Hv) [16] based on previous studies. Details of acquisition and preprocessing of $\left[{ }^{11} \mathrm{C}\right] \mathrm{PiB}-\mathrm{PET},\left[{ }^{18} \mathrm{~F}\right] \mathrm{FDG}-\mathrm{PET}$, and MRI and the definition of each $\mathrm{AD}$ imaging biomarker are provided in the Supplementary Methods and Supplementary Table S2. 


\section{$A D$ gene panel, sequencing, and variant calling}

The 132 genes were selected according to one of the following criteria: (1) genes from previous AD GWAS ( $n=$ 32) [6-9], (2) genes with mutations reported to be causative for familial AD in the OMIM database $(n=$ 19), (3) genes with biological relevance in $A D$ in the KEGG pathway (hsa05010) $(n=53)$, or (4) manually selected genes with potential relevance in $\mathrm{AD}(n=28)$ (Supplementary Table S3). The AD gene panel was designed using the Ion AmpliSeq Designer (http://ampliseq.com) and contained 5049 amplicons with sizes ranging from 125 to $275 \mathrm{bp}$. The target regions included UTR and coding exons with 10 bases of padding sequences, totaling $1.46 \mathrm{Mb}$ and covering $96 \%$ of the targeted intervals. AD-targeted panel sequencing was performed with an Ion Proton sequencer (Supplementary Methods, Supplementary Fig. S1 and Supplementary Table S4). The reads were aligned to hg19 and SNVs were called using Torrent Suite v3.4.2. Variants with < $20 \times$ coverage, located in homopolymer repeats, or covered $<90 \%$ of the samples were excluded. After applying the variant filtering criteria established in our pilot experiments (Supplementary Methods and Supplementary Fig. S1), 5391 variants remained.

\section{Association tests}

Both continuous and categorical variables indicating in vivo $A D$ pathologies were normalized and used as dependent variables to identify common variants (allele frequency $(\mathrm{AF})>0.05)$ associated with bioimaging markers (Supplementary Methods and Supplementary Table S2). Age, sex, and the number of APOE4 alleles were used as covariates. Significant associations were defined at an uncorrected $P<0.001$, following the previous studies [20-22], and the Benjamini and Hochberg method was used for multiple test correction for $A P O E$ variant association tests (Supplementary Table S5). Among the rare variants $(\mathrm{AF}<0.05)$, loss-of-function (LoF) variants and missense variants with strong evolutionary conservation were selected for gene-based association analyses. $\mathrm{R}$ (version 3.5.1) was used to conduct Fisher's exact test with the five binominal neuroimaging traits (Supplementary Table S2) and cognitive impairment, and rare variant enrichment across each gene was adjusted by length. For $A P O E$ variants, variants with $\mathrm{AF}>0.1$ were selected and tested for association, after adjusting for age and sex. For conditional analysis, a quantitative imaging trait was used as a covariate in an association test of $A P O E$ variants and $\mathrm{AD}$ susceptibility.

\section{Voxel-based analysis of multi-modal brain imaging}

To identify regional $\mathrm{PiB}$ uptake differences, Statistical Parametric Mapping 12 (SPM12; http://www.fil.ion.ucl. ac.uk/spm) was used for the exploratory voxel-based analysis of $\left[{ }^{11} \mathrm{C}\right] \mathrm{PiB}-\mathrm{PET}$. Similarly, voxel-based analyses of $\left[{ }^{18} \mathrm{~F}\right]$ FDG-PET and T1 MRI were performed to visualize the differences of regional cerebral glucose metabolism or gray matter (GM) density by the presence of variants associated with $\mathrm{AD}-\mathrm{Cm}, \mathrm{PCC}-\mathrm{Cm}, \mathrm{AD}-\mathrm{Ct}$, or Hv. Additional details of the voxel-wise analysis of common variants and genes with rare variants are provided in the Supplementary Methods.

\section{Results}

Establishment of the AD panel, quality assessment, and variant calling strategy

The overall study design and documented in vivo pathology biomarkers are represented in Fig. 1a, b. The clinical and demographic characteristics of the 557 participants are described in Supplementary Table S1. The mean age of the participants is 67.4 years, and $57.8 \%$ are women. The frequency of the APOE4 allele in each diagnostic group indicated a strong disease association (i.e., 9.3\% and $35.7 \%$ for $\mathrm{CN}$-old and dementia groups, respectively). The mean coverage depth of the $\mathrm{AD}$ target sequencing runs was $436.8 \times$, and $97.8 \%$ of the targeted bases were covered more than $20 \times$, ensuring high variant sensitivity (Supplementary Table S4).

We developed an in-house SNV calling method for ion proton sequencing data. Prior to data production, we optimized the variant calling pipeline through a series of pilot experiments and demonstrated $92.4 \%$ sensitivity and $93.5 \%$ specificity (Supplementary Fig. S1). To further validate the quality of the 5391 called variants, principal component analysis (PCA) of the KBASE cohort was performed with individuals from the $1000 \mathrm{Ge}$ nomes Project, which showed that our cohort coclustered with the East Asian group (Fig. 1c). A strong correlation of AFs between our cohort and $1000 \mathrm{Ge}-$ nomes East Asian was observed (Pearson's $r=0.99$ [95\% CI, 0.989-0.991]). The majority of the variants (79.2\%) are rare $(\mathrm{AF}<0.05)$, and $45.2 \%$ were singletons (seen once in our cohort; Fig. 1d). The majority of the variants (88.9\%) with an AF $>0.001$ are already listed in the 1000 Genomes, The Genome Aggregation Database (gnomAD), and the Korean Variant Archive (KOVA) [23-25]. The variants are composed of 31.5\% UTR, $1.9 \%$ intergenic, $33.7 \%$ intronic, and $32.9 \%$ coding region locations (Fig. 1e). Each individual carried an average of 369.0 heterozygous variants, 295.4 homozygous variants, and 4.4 singletons. The mean ratio of missense-to-silent variants was 0.44 , and the mean ratio of transition-to-transversion variants was 2.83 per individual, comparable to other large-scale genome data sets [26].

\section{Common variant association test}

Tests that evaluated the association between common variants and $\mathrm{A} \beta$ deposition, $\mathrm{AD}-\mathrm{Cm}, \mathrm{PCC}-\mathrm{Cm}, \mathrm{AD}-\mathrm{Ct}$, 


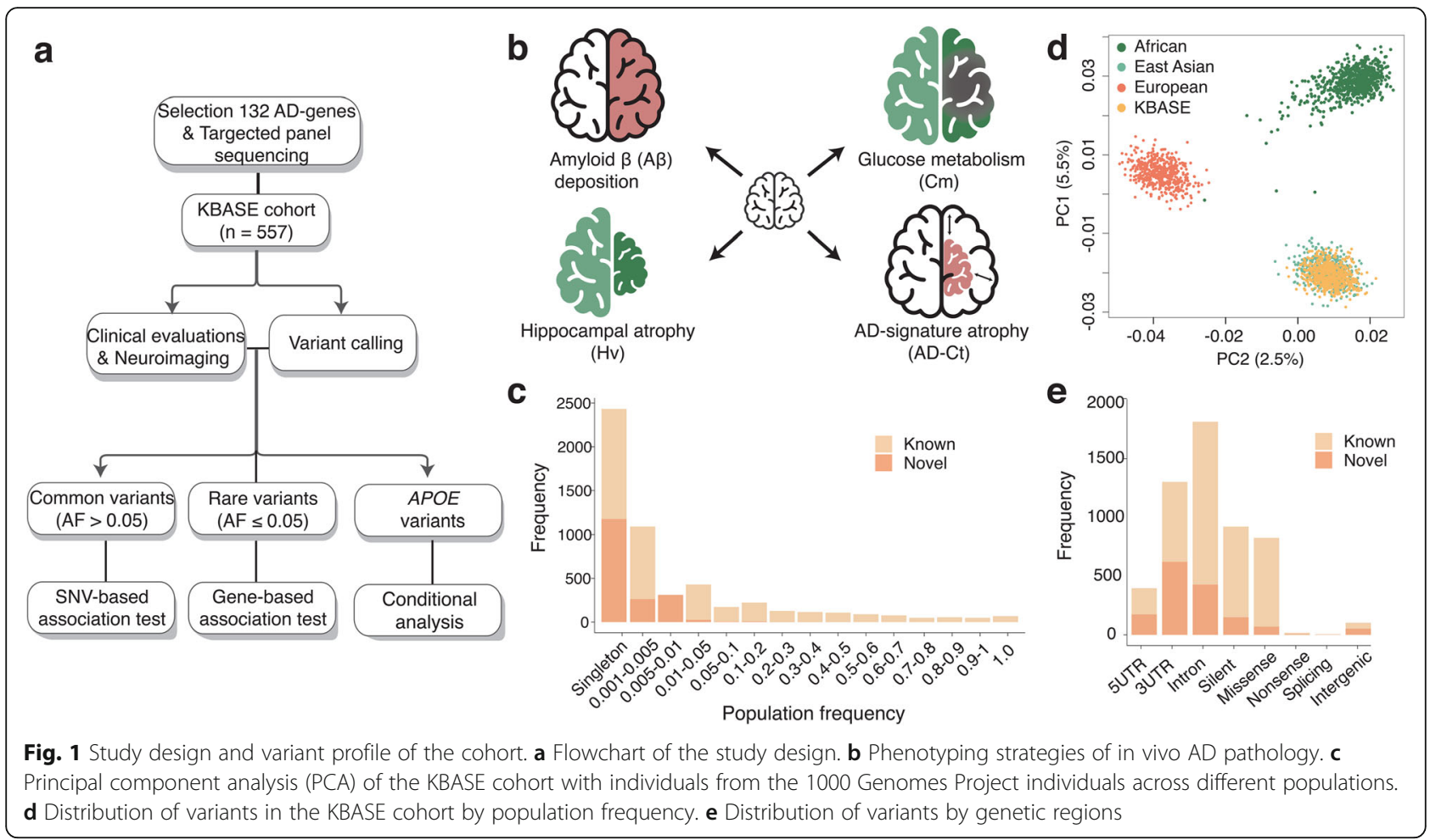

and $\mathrm{Hv}$ revealed seven loci with strong associations, of which six are novel (Table 1 ). The $\mathrm{CN}$-ym group was excluded from the test, as this group was entirely negative for the in vivo $\mathrm{AD}$ imaging biomarkers regardless of genotype.

Variants in GORASP1 (rs28362644; odds ratio [OR] 0.46 [0.29-0.72]; $\left.P=7.0 \times 10^{-4}\right), \quad M A D D$ (rs2290149; OR $\left.2.74 \quad[1.61-4.68] ; \quad P=2.0 \times 10^{-4}\right)$, and PIWIL1 (rs10848087; regression coefficient $[\beta] 0.14$ [0.060.22 ]; $P=5.1 \times 10^{-4}$ ) were significantly associated with A $\beta$ deposition (Table 1; Fig. 2a; Supplementary Fig. S2a). Comparison with voxel-based imaging data $(P<$ $0.01, k=1497)$ revealed that carriers of $M A D D$ rs2290149 displayed greater $A \beta$ deposition mainly in the bilateral, lateral, and medial frontal cortices; cingulate; precuneus; and lateral temporal and inferior parietal regions. PIWIL1-rs10848087 non-carriers showed diffuse $A \beta$ accumulation in the bilateral cerebral cortices including the frontal, temporal, parietal, occipital lobes and basal ganglia (Fig. 2a). Three SNVs near GORASP1 were identified, located in the same linkage disequilibrium (LD) block $\left(R^{2}=0.98 ; D^{\prime}=\right.$ 0.99), and the most robust SNP, rs28362644, was used for imaging analysis (Table 1). GORASP1-rs28362644 carriers showed less $A \beta$ deposition in the posterior hippocampal and parahippocampal regions, as well as retrosplenial and precuneus regions at a more lenient threshold compared to non-carriers $(P<0.05, \quad k=$ 5058; Supplementary Fig. S2a).
Two novel significant associations with $\mathrm{AD}-\mathrm{Cm}$ were observed: PSEN2-rs75733498 (OR 0.39 [0.23-0.63]; $P=$ $\left.1.8 \times 10^{-4}\right)$ and NME8-rs2722372 $(\beta-0.04$ [ -0.06 to 0.018 ]; $P=7.6 \times 10^{-4}$ ) (Table 1; Fig. 2b; Supplementary Fig. S2b). Subsequent voxel-based FDG-PET analysis revealed that PSEN2-rs75733498 non-carriers had reduced glucose metabolism levels in the bilateral frontotemporo-parietal cortices $(P<0.01, k=1497$; Fig. $2 b)$. On the other hand, NME8-rs2722372 carriers displayed diffuse cerebral glucose hypometabolism in the bilateral fronto-temporo-parietal cortices and subcortical structures, such as the thalamus and basal ganglia (Fig. 2b). An association between AD-Cm and NME8-rs2722372 in the PCC was also observed.

PSEN1-rs7523 (OR 0.39 [0.25-0.60]; $P=1.7 \times 10^{-5}$ ) and PIWIL1-rs10848087 $(\beta-0.07$ [ -0.11 to -0.03$] ; P=$ $2.9 \times 10^{-4}$ ) were associated with AD-Ct (Table 1; Fig. 2c; Supplementary Fig. S2c). Exploratory voxel-based analysis of T1 MRI demonstrated that PSEN1-rs7523 noncarriers showed reduced cortical thicknesses as compared to carriers in the inferior frontal, orbitofrontal, and basal ganglia $\left(P=2.5 \times 10^{-5}, k=1497\right.$; Fig. $\left.2 \mathrm{~d}\right)$.

Finally, CASS4-rs3746625 $(\beta$ 0.54 $[0.26-0.82] ; P=$ $\left.1.7 \times 10^{-4}\right)$ and PIWIL1-rs10848087 $(\beta-0.40[-0.61$ to $-0.19] ; \quad P=2.3 \times 10^{-4}$ ) strongly correlated with $\mathrm{Hv}$ (Table 1; Fig. 2d; Supplementary Fig. S2c). There are also three other significant SNVs in CASS4 that are located in the same LD block with $\operatorname{rs3746625}\left(R^{2}=1.00 ; D^{\prime}=\right.$ 1.00). Brain images showed that $G M$ atrophy was 
Table 1 List of common variants that significantly associated with brain imaging features $\left(P<1.0 \times 10^{-3}\right)$

\begin{tabular}{|c|c|c|c|c|c|c|c|}
\hline AD imaging biomarker & Data type & Chr:position (hg19) & dbSNP ID & Gene $^{a}$ & $\mathrm{PF}^{\mathbf{b}}$ & Previously reported & $P$ \\
\hline \multicolumn{8}{|c|}{ I. Cerebral amyloid- $\beta$ accumulation measured by PiB-PET } \\
\hline \multirow[t]{5}{*}{$A \beta$ deposition } & \multirow[t]{4}{*}{ Bin. $^{c}$} & chr3:39138840 & rs3732377 & GORASP1 & 0.237 & Novel & $9.32 \times 10^{-4}$ \\
\hline & & chr3:39139776 & rs1109643 & GORASP1 & 0.161 & Novel & $9.79 \times 10^{-4}$ \\
\hline & & chr3:39149277 & rs28362644 & GORASP1 & 0.162 & Novel & $7.02 \times 10^{-4}$ \\
\hline & & chr11:47345916 & rs2290149 & MADD & 0.082 & Novel & $2.02 \times 10^{-4}$ \\
\hline & Quant. $^{\text {d }}$ & chr12:130839165 & rs10848087 & PIWIL1 & 0.105 & Novel & $5.05 \times 10^{-4}$ \\
\hline \multicolumn{8}{|c|}{ II. Glucose metabolism levels measured by FDG-PET } \\
\hline \multirow[t]{2}{*}{$\mathrm{AD}-\mathrm{Cm}$} & Bin. & chr1:227077809 & rs75733498 & PSEN2 & 0.083 & Novel & $1.75 \times 10^{-4}$ \\
\hline & Quant. & chr7:37890267 & rs2722372 & NME8 & 0.191 & Novel & $7.63 \times 10^{-4}$ \\
\hline PCC-Cm & Quant. & chr7:37890267 & rs2722372 & NME8 & 0.191 & Novel & $5.71 \times 10^{-4}$ \\
\hline \multicolumn{8}{|c|}{ III. Cortical thickness measured by MRI } \\
\hline \multirow[t]{2}{*}{$A D-C t$} & Bin. & chr14:73686944 & rs7523 & PSEN1 & 0.161 & Novel & $1.74 \times 10^{-5}$ \\
\hline & Quant. & chr12:130839165 & rs10848087 & PIWIL1 & 0.105 & Novel & $2.94 \times 10^{-4}$ \\
\hline \multicolumn{8}{|c|}{ IV. Hippocampal volume reduction measured by MRI } \\
\hline \multirow[t]{5}{*}{$\mathrm{Hv}$} & Quant. & chr20:55033476 & rs3746623 & CASS4 & 0.945 & Novel & $1.73 \times 10^{-4}$ \\
\hline & & chr20:55033647 & rs3746625 & CASS4 & 0.946 & Novel & $1.73 \times 10^{-4}$ \\
\hline & & chr20:55033713 & rs3746626 & CASS4 & 0.946 & Novel & $1.73 \times 10^{-4}$ \\
\hline & & chr20:55033856 & rs4811697 & CASS4 & 0.946 & Known [27] & $3.42 \times 10^{-4}$ \\
\hline & Both & chr12:130839165 & rs10848087 & PIWIL1 & 0.105 & Novel & $4.24 \times 10^{-4}$ \\
\hline
\end{tabular}

${ }^{a}$ The most closely located gene from each variant

${ }^{b}$ Population frequency in the KBASE cohort

${ }^{c}$ Categorical variable trait transformed from normalized neuroimaging data by each criterion

${ }^{\mathrm{d}}$ Quantitative normalized neuroimaging variable trait

reduced in rs3746625 carriers than non-carriers, particularly in the bilateral medial temporal regions including the hippocampi $(P<0.01, k=1497$; Fig. $2 d)$. To elucidate the functional implication of the CASS4 and PIWIL1 variants in the brain, ChIP-seq data from the ENCODE were utilized [28]. Notably, H3K27me3 signals, which mark heterochromatin, encompass the variants specifically in the hippocampus, but not in other parts of the brain or other organs, consistent with the results of voxel-based analysis of MRI (Supplementary Fig. S3).

The cumulative effect of common variants associated with the imaging biomarkers was evaluated by correlating $\mathrm{AD}$ risk score, defined as the weighted sum of the effect sizes of common variants associated with imaging features and the proportion of individuals with cognitive impairment (i.e., MCI or ADD; Supplementary Fig. S4). Common SNVs associated with each imaging biomarker $(P<0.05)$ explained $27-58 \%$ of cognitive impairment and $76 \%$ when used in combination.

\section{Effect of APOE variants}

The APOE4 allele possesses the strongest association with AD susceptibility [8], but comprehensive analysis of its impact on structural and functional changes in a large number of aging brains remains understudied. In addition, our study design allowed association assessment of other $A P O E$ variants. Therefore, we sought to identify association between $A P O E$ common variants and the imaging features. Rare variants were not detected. We tested three common variants with an $\mathrm{AF}>0.1$. Two variants, rs429358 and rs769449, are located in the same LD block $\left(R^{2}=0.73, D^{\prime}=0.95\right.$; Fig. 3a) and are strongly correlated with all five imaging traits (Table 2; Fig. 3b). An additional variant (rs405509) located in the promoter is in weak LD with rs429358 $\left(R^{2}=0.05, D^{\prime}=1.00\right)$ and $\operatorname{rs769449}\left(R^{2}=0.06, D^{\prime}=0.96\right.$; Fig. 3a). rs405509 possessed minimal association with the brain features (Table 2), with the association specifically residing in the 3 '-portion of the gene.

Conditional analysis was performed to test if the influence of these $A P O E$ variants on neuroimaging features is causal to ADD risk (Table 2). Adjusting for each brain imaging feature effectively reduced the association between the two SNVs (rs429358 and rs769449) and AD susceptibility by approximately $10^{5}$ - to $10^{8}$-fold in $P$ values. This result suggests that the $A P O E$ variants modulate neuroimaging features that contributes to $\mathrm{AD}$ susceptibility. Conversely, the significance of another variant (rs405509) did not change after conditioning, indicating that brain imaging features are independent of its association with AD susceptibility. 

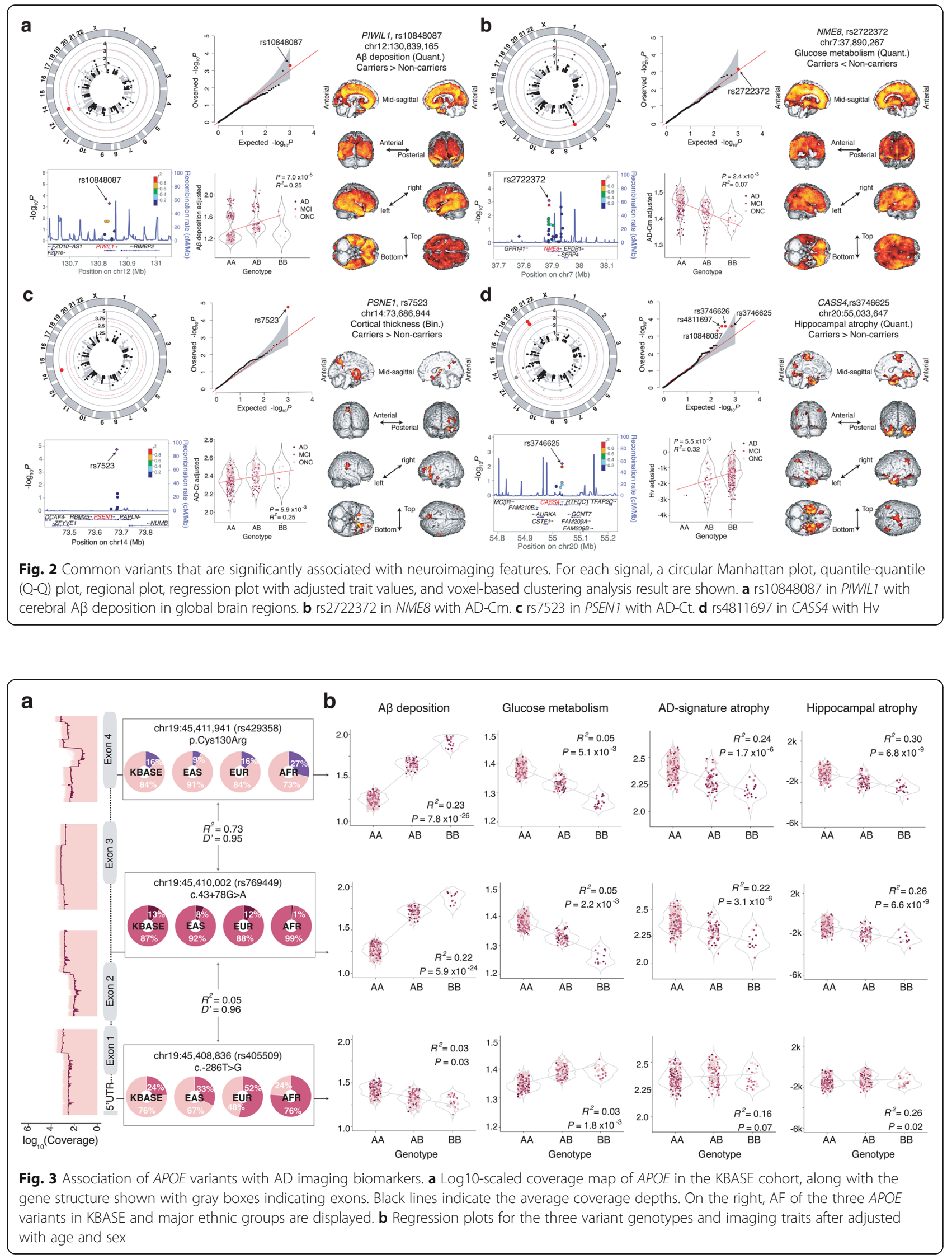
Table 2 Effects of common APOE SNVs on imaging biomarkers and conditional analysis of the SNVs controlling for each imaging biomarkers

\begin{tabular}{|c|c|c|c|c|c|c|}
\hline \multirow[t]{2}{*}{ APOE SNP } & \multirow{2}{*}{$\begin{array}{l}\text { Imaging } \\
\text { biomarkers }\end{array}$} & \multicolumn{3}{|c|}{ Association (Quant.) } & \multicolumn{2}{|c|}{ Cognitive function association } \\
\hline & & $\beta$ & $P$ & $P_{\text {adj }}$ & Unconditioned & Controlling for imaging biomarker (Quant.) \\
\hline \multirow[t]{5}{*}{ rs429358 (chr19:45411941) } & A $\beta$ deposition & 0.37 & $2.85 \times 10^{-27}$ & $2.89 \times 10^{-24}$ & $2.03 \times 10^{-9}$ & 0.047 \\
\hline & $\mathrm{AD}-\mathrm{Cm}$ & -0.06 & $5.61 \times 10^{-6}$ & $2.85 \times 10^{-3}$ & & $2.18 \times 10^{-5}$ \\
\hline & PCC-Cm & -0.10 & $3.07 \times 10^{-10}$ & $3.12 \times 10^{-7}$ & & $3.16 \times 10^{-3}$ \\
\hline & $\mathrm{AD}-\mathrm{Ct}$ & -0.11 & $8.70 \times 10^{-13}$ & $8.80 \times 10^{-10}$ & & $2.82 \times 10^{-3}$ \\
\hline & $\mathrm{H} v$ & -0.78 & $2.23 \times 10^{-18}$ & $2.25 \times 10^{-15}$ & & 0.150 \\
\hline \multirow[t]{5}{*}{ rs769449 (chr19:45410002) } & A $\beta$ deposition & 0.39 & $1.51 \times 10^{-25}$ & $7.65 \times 10^{-23}$ & $7.29 \times 10^{-8}$ & 0.260 \\
\hline & $\mathrm{AD}-\mathrm{Cm}$ & -0.06 & $2.78 \times 10^{-6}$ & $2.82 \times 10^{-3}$ & & $3.08 \times 10^{-3}$ \\
\hline & PCC-Cm & -0.10 & $8.58 \times 10^{-9}$ & $4.36 \times 10^{-6}$ & & 0.052 \\
\hline & $\mathrm{AD}-\mathrm{Ct}$ & -0.11 & $2.16 \times 10^{-9}$ & $1.09 \times 10^{-6}$ & & 0.022 \\
\hline & $\mathrm{H} v$ & -0.73 & $3.72 \times 10^{-13}$ & $1.88 \times 10^{-10}$ & & 0.210 \\
\hline \multirow[t]{5}{*}{ rs405509 (chr19:45408836) } & $A \beta$ deposition & -0.09 & $5.78 \times 10^{-3}$ & 0.84 & $1.94 \times 10^{-3}$ & 0.020 \\
\hline & $\mathrm{AD}-\mathrm{Cm}$ & 0.03 & $3.15 \times 10^{-3}$ & 0.40 & & 0.029 \\
\hline & PCC-Cm & 0.03 & 0.017 & 0.83 & & 0.032 \\
\hline & $\mathrm{AD}-\mathrm{Ct}$ & 0.02 & 0.16 & 0.99 & & 0.011 \\
\hline & $\mathrm{HV}$ & 0.14 & 0.089 & 0.96 & & 0.017 \\
\hline
\end{tabular}

\section{Gene-level analysis for rare variants}

To investigate the associations of rare variants with $\mathrm{AD}$ imaging biomarkers and cognitive impairment, we collected functional rare variants $(\mathrm{AF}<0.05$, LoF or evolutionary conserved missense variants). A total of eight genes appeared to be significant at a gene-level analysis $(P<0.05$; Fig. 4; Supplementary Table S6 and Supplementary Fig. S5). Variants in $L P L$ were detected only in cases with high $\mathrm{A} \beta$ deposition ( $n=3, P=0.03$; Fig. $4 \mathrm{a})$. No rare variants were significantly associated with $\mathrm{AD}-\mathrm{Cm}$ hypometabolism, but FERMT2 (OR 2.37 [1.07-5.61]; $P=0.02$ ) and NFAT5 (OR 4.24 [1.15-23.32]; $P=0.02$ ) showed significant enrichment for the presence of PCC hypometabolism (Table. S5). DSG2 showed significant enrichment in controls with normal AD-Ct and $\mathrm{Hv}$ (OR 0.17 [0.020.89], $P=0.02$, and OR 0.0 [0.00-1.02], $P=0.03$; Fig. 4b; Supplementary Fig. S5a). Interestingly, the observed rare variants were mainly located in the sites that bind calcium ions or other proteoglycans, such as $N$-acetylglucosamine (Supplementary Fig. S6c). Also, functional variants in ITPR1 were observed only in controls with normal Hv $(n=9$; Fig $4 \mathrm{c})$. These results demonstrate novel associations between rare variants and brain features that can be documented by imaging techniques.

\section{Discussion}

We investigated the associations between genetic variants and various brain features that are accessible via imaging techniques. We identified a group of variants that are linked to $\mathrm{A} \beta$ deposition, $\mathrm{AD}-\mathrm{Cm}, \mathrm{PCC}-\mathrm{cm}, \mathrm{AD}-$ $\mathrm{Ct}$, and $\mathrm{Hv}$ (Fig. 1b). Our results bolster the role of
$A P O E$ alleles in contributing to $\mathrm{AD}$ pathogenesis susceptibility.

The association analysis of common variants led to the discovery of seven significant associations with AD brain imaging biomarkers. Particularly, we observed an association between CASS4 common variants and Hv (Table 1). Previous association studies suggested that CASS4, encoding a cytoskeletal protein that provides a physical connection with the extracellular matrix, contributes to the risk of ADD [8, 27]. Our observation implies that the previously reported association between CASS4 and ADD may be through neurodegeneration of the hippocampus via an A $\beta$-independent pathway. Voxel-wise analysis of MRI demonstrated a region-specific GM atrophy in the bilateral hippocampi and adjacent regions in CASS4 variant carriers (Fig. 2d). The presence of hippocampus-specific H3K27me3 signals (Supplementary Fig. S3a) suggests that these variants are related to a region-specific epigenetic modification in the hippocampus, although future studies will be necessary to clarify the function of these CASS4 variants.

PIWIL1 common variants are related to both $A \beta$ deposition and atrophy in the $\mathrm{AD}$-signature region and the hippocampus (Table 1 and Supplementary Table S7). Voxel-wise analysis of PiB-PET consistently revealed that PIWIL1 variant carriers displayed greater levels of cerebral $A \beta$ deposition as compared to non-carriers, and regional GM atrophy mainly in the bilateral temporal and PC-PCC areas and medial and inferior frontal regions, which were reported to be vulnerable to the $\mathrm{AD}$ process [19]. PIWIL1 is a member of the PIWI subfamily 


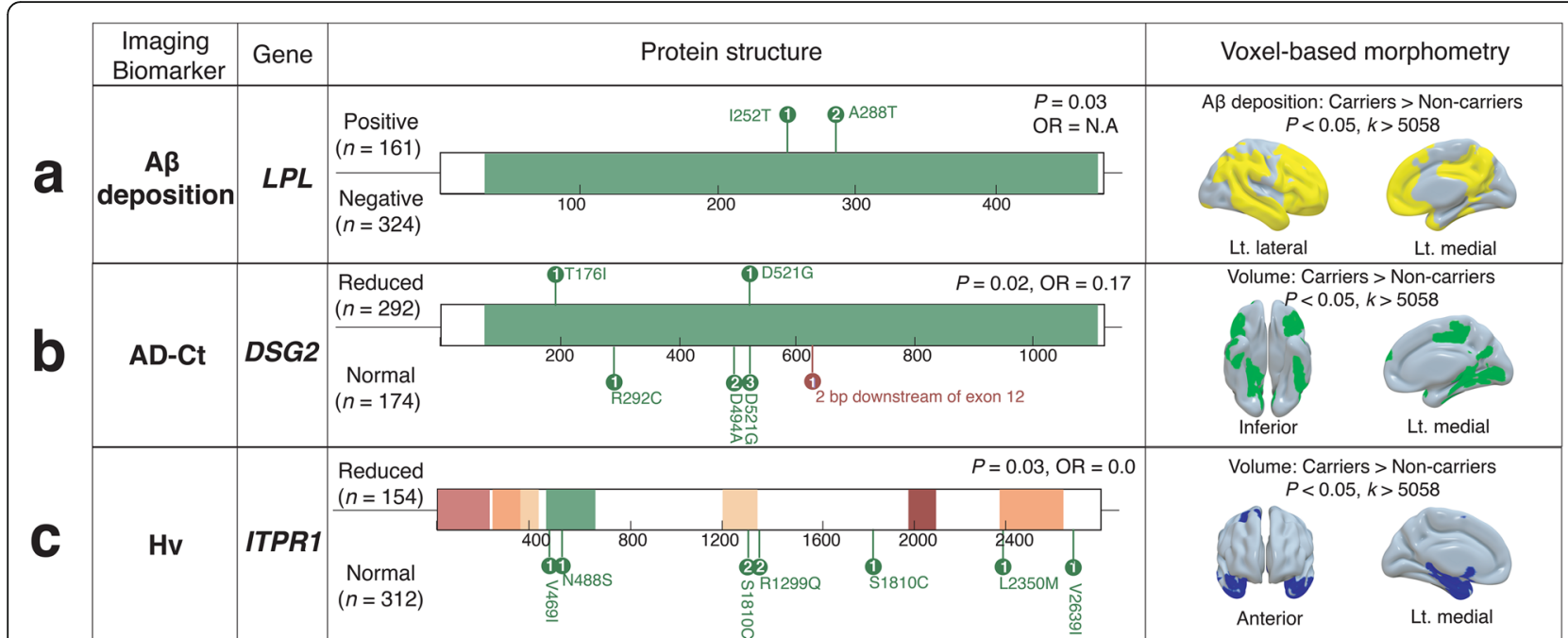

Fig. 4 Genes with rare variants that are significantly associated with in vivo AD pathologies. Observed rare functional variants in the case or control groups defined by each clinical parameter are shown for each gene. a LPL with A 3 deposition in the global brain regions. b DSG2 with AD-signature cortical thickness. c ITPR1 with hippocampal volume. The right panel displays the exploratory voxel-based analyses of brain imaging to demonstrate the regional pattern differences in $\mathrm{AD}$ imaging biomarker phenotype between carriers and non-carriers

that plays important roles in stem cell self-renewal, RNA silencing, translational regulation, and neuron development [29], which potentially implicates multiple aspects of brain degeneration. Moreover, the PIWIL1 variants also co-localize with a brain-specific H3K27me3 signal (Supplementary Figure S3b), which may provide insight into the underlying function of PIWIL1 in neurodegeneration of $\mathrm{AD}$-vulnerable regions. Additionally, significant associations between common variants in PSEN1 and PSEN2 and neurodegeneration biomarkers of $\mathrm{AD}$ (i.e., PSEN1-rs7523 with AD-Ct and PSEN2-rs75733498 with $\mathrm{AD}-\mathrm{Cm}$ ) were observed. Pathogenic rare variants in these genes cause early-onset familial AD. These genes encode major components of the $\gamma$-secretase of APP synthesis and proteolysis, leading to $A \beta$ production [30]. It is not clear how these variants are associated with AD-type neurodegeneration, but this observation demonstrates that dysregulation of the genes confers susceptibility to $\mathrm{AD}$ via alterations of regional cerebral glucose metabolism or regional cortical thickness, which demonstrates their versatile roles in $\mathrm{AD}$ pathogenesis beyond altering $A \beta$ production.

Unlike common or rare variants with association, we found associations between $A P O E$ variants and all brain imaging features of $\mathrm{AD}$. This finding is consistent with previous reports [31-35], conferring a partial explanation for the genetic link between APOE and AD susceptibility. An association test of a variant in the other LD block revealed a marginal association, suggesting a specific functional association between the cell surface receptor binding region and the imaging features. Conditional analysis demonstrated a causal relationship between the imaging features and the association of $A P O E$ variants with AD susceptibility (Table 2).

The gene-level rare variant association test revealed several genes previously related to $\mathrm{AD}$ brain features with cognitive impairment. Notably, carriers of DSG2 rare variants harbor increased $\mathrm{AD}-\mathrm{Ct}, \mathrm{Hv}$, and cognitive function, suggesting that these variants play protective roles in neurodegeneration in AD-related regions (Fig $4 \mathrm{~b}$ and Supplementary Fig. S5a). DSG2 encodes a calciumbinding glycoprotein components of the desmosome that binds to plaque proteins and intermediate filaments [36] and was reported to confer $\mathrm{AD}$ risk based on GWAS [8]. Although a molecular link explaining this association remains unclear, we observed that the variants were located in calcium ion or proteoglycan binding sites (Supplementary Fig. S5c). These sites are evolutionally conserved, and the binding of these molecules is essential for protein function [37].

The KBASE protocol ensures unified subject assessment and standardization of all samples and data collection, processing, and quality control. It enables multi-trait analysis of genetic loci, explicitly targeting each in vivo $\mathrm{AD}$ pathology rather than relying on clinical diagnoses with limited accuracy.

\section{Strengths and limitations}

All individuals enrolled in the KBASE cohort underwent careful clinical and genetic characterization. The KBASE protocol adopts unified subject assessment, standardization of all imaging, biofluid, DNA and RNA data collection, and processing followed by meticulous data quality control. We analyzed variants of wide range of allele frequencies on 
AD-associated genes. Therefore, 132 AD-associated genes for LOAD were covered for genetic variant associations with the AD brain imaging features. Our next step will be to scrutinize the molecular biological mechanisms of variants and their functions on AD pathology.

Several limitations of our study warrant discussions. First, we tried to replicate our results using the ADNI database, which includes unified clinical information, brain imaging data, and genomic data of participants. We adjusted raw brain imaging data of ADNI database using our methodology and conducted association analyses. However, replication was not feasible due to the allele frequency differences originated from the ethnic discrepancies and differences in phenotyping methods, except the APOE SNVs. We also collected a large-scale elderly Korean cohort $(n=4683)$ and conducted a sequencing analysis using our customized targeted LOAD gene panel. Associations between FDG uptake and two novel SNPs were replicated-rs75733498 on PSEN2 $(P=$ $\left.1.6 \times 10^{-4}\right)$ and rs2722372 on NME8 $\left(P=8.6 \times 10^{-3}\right)$-although this cohort lacks brain imaging assessment and cognitive impairment trait was accessible.

\section{Conclusions}

Here, we explored the KBASE data and conducted integrative analysis that revealed novel associations between AD-related gene variants and various brain features. Thus, scrutinizing the biological mechanisms of genetic variants and their functions in $\mathrm{AD}$ pathology by interrogating multiple aspects of brain morphology and in vivo $\mathrm{AD}$ pathologies will lead to a better understanding of the mechanism of $\mathrm{AD}$ pathogenesis.

\section{Supplementary Information}

Supplementary information accompanies this paper at https://doi.org/10. 1186/s13195-020-00722-2.

Additional file 1. Supplementary methods, tables, and figures.

\section{Acknowledgements}

We thank the participants and members of the KBASE consortium. Professor Han Buhm provided us with critical comments.

The coinvestigators of the KBASE Research Group are listed elsewhere (http://kbase.kr).

\section{Authors' contributions}

Conceptualization: MC, IM-J, and DYL. Formal analysis: JS and MSB. Methodology: JS and MC. Genome data acquisition: JS, J-CP, S-HH, JB, IM-J, and MC. Clinical data acquisition: MSB, DY, JHL, SYJ, SAS, YKK, KMK, C-HS, GJ, and DYL. Supervision: MC and DYL. Writing—original draft: JS and MC. Writing, review, and editing: JS, MSB, DYL, and MC. The authors read and approved the final manuscript.

\section{Funding}

The study was supported by grants from the National Research Foundation of Korea (2014M3C7A1046049 and 2018M3C9A5064708 for Choi M and 2014M3C7A1046042 for Lee DY) and grants from the Ministry of Health and Welfare of Korea (HI18C0630 for Mook-Jung IH and Lee DY, and HI19C0149 for Lee DY).

\section{Availability of data and materials}

All data needed to evaluate the conclusions in the paper are present in the paper. Additional data related to this paper may be requested from the authors.

\section{Ethics approval and consent to participate}

The Institutional Review Boards of Seoul National University Hospital and SMGSNU Boramae Medical Center approved the study (1401-027-547 and 26-201560). This study was conducted in accordance with the recommendations of the current version of the Declaration of Helsinki. All participants and/or their legal representatives provided written informed consent.This action is appropriate.

\section{Consent for publication}

All authors consented for the publication of the manuscript.

\section{Competing interests}

The authors declare no conflicts of interest.

\section{Author details}

${ }^{1}$ Department of Biomedical Sciences, Seoul National University College of Medicine, Seoul, Republic of Korea. ${ }^{2}$ Department of Neuropsychiatry, Seoul National University Bundang Hospital, Gyeonggi, Republic of Korea. ${ }^{3}$ Institute of Human Behavioral Medicine, Medical Research Center Seoul National University, Seoul, Republic of Korea. ${ }^{4}$ Department of Neuropsychiatry, National Center for Mental Health, Seoul, Republic of Korea. ${ }^{5}$ Department of Psychiatry, Chungnam National University Hospital, Daejeon, Republic of Korea. ${ }^{6}$ Department of Nuclear Medicine, SMG-SNU Boramae Medical Center, Seoul, Republic of Korea. ${ }^{7}$ Department of Radiology, Seoul National University Hospital, Seoul, Republic of Korea. ${ }^{8}$ Department of

Neuropsychiatry, Seoul National University Hospital, Seoul, Republic of Korea. ${ }^{9}$ Department of Biochemistry, Seoul National University College of Medicine, Seoul, Republic of Korea. ${ }^{10}$ Department of Medicine, Pusan National University, Busan, Republic of Korea. ${ }^{11}$ Department of Psychiatry, Seoul National University College of Medicine, Seoul, Republic of Korea.

Received: 2 July 2020 Accepted: 6 November 2020

Published online: 19 November 2020

\section{References}

1. Gatz M, Reynolds CA, Fratiglioni L, Johansson B, Mortimer JA, Berg S, et al. Role of genes and environments for explaining Alzheimer disease. Arch Gen Psychiatry. 2006;63(2):168-74.

2. Chouraki V, Seshadri S. Chapter five - Genetics of Alzheimer's disease. In: Friedmann T, Dunlap JC, Goodwin SF, editors. Advances in Genetics. 87: Academic Press; 2014. p. 245-94.

3. Bekris LM, Yu CE, Bird TD, Tsuang DW. Genetics of Alzheimer disease. J Geriatr Psychiatry Neurol. 2010;23(4):213-27.

4. Liu CC, Liu CC, Kanekiyo T, Xu H, Bu G. Apolipoprotein E and Alzheimer disease: risk, mechanisms and therapy. Nat Rev Neurol. 2013;9(2):106-18.

5. Farrer LA, Cupples LA, Haines JL, Hyman B, Kukull WA, Mayeux R, et al. Effects of age, sex, and ethnicity on the association between apolipoprotein E genotype and Alzheimer disease. A meta-analysis. APOE and Alzheimer Disease Meta Analysis Consortium. JAMA. 1997;278(16):1349-56.

6. Harold D, Abraham R, Hollingworth P, Sims R, Gerrish A, Hamshere ML, et al. Genome-wide association study identifies variants at CLU and PICALM associated with Alzheimer's disease. Nat Genet. 2009;41(10):1088-93.

7. Hollingworth P, Harold D, Sims R, Gerrish A, Lambert JC, Carrasquillo MM, et al. Common variants at ABCA7, MS4A6A/MS4A4E, EPHA1, CD33 and CD2AP are associated with Alzheimer's disease. Nat Genet. 2011;43(5):429-35.

8. Lambert JC, Ibrahim-Verbaas CA, Harold D, Naj AC, Sims R, Bellenguez C, et al. Meta-analysis of 74,046 individuals identifies 11 new susceptibility loci for Alzheimer's disease. Nat Genet. 2013;45(12):1452-8.

9. Seshadri S, Fitzpatrick AL, Ikram MA, DeStefano AL, Gudnason V, Boada M, et al. Genome-wide analysis of genetic loci associated with Alzheimer disease. JAMA. 2010;303(18):1832-40.

10. Bis JC, Jian X, Kunkle BW, Chen Y, Hamilton-Nelson KL, Bush WS, et al. Whole exome sequencing study identifies novel rare and common Alzheimer's-Associated variants involved in immune response and transcriptional regulation. Mol Psychiatry. 2020;25(8):1859-75. 
11. Khera AV, Chaffin M, Wade KH, Zahid S, Brancale J, Xia R, et al. Polygenic prediction of weight and obesity trajectories from birth to adulthood. Cell. 2019;177(3):587-96 e9.

12. Wainschtein $P$, Jain DP, Yengo $L$, Zheng $Z$, Cupples LA, Shadyab $A H$, et al. Recovery of trait heritability from whole genome sequence data. bioRxiv; 2019. https://doi.org/10.1101/588020.

13. Byun MS, Yi D, Lee JH, Choe YM, Sohn BK, Lee JY, et al. Korean brain aging study for the early diagnosis and prediction of Alzheimer's disease: methodology and baseline sample characteristics. Psychiatry Investig. 2017; 14(6):851-63.

14. Jack CR Jr, Albert MS, Knopman DS, MCKhann GM, Sperling RA, Carrillo MC, et al. Introduction to the recommendations from the National Institute on Aging-Alzheimer's Association workgroups on diagnostic guidelines for Alzheimer's disease. Alzheimers Dement. 2011;7(3):257-62.

15. Jack CR Jr, Knopman DS, Jagust WJ, Shaw LM, Aisen PS, Weiner MW, et al. Hypothetical model of dynamic biomarkers of the Alzheimer's pathological cascade. Lancet Neurol. 2010;9(1):119-28.

16. Jack CR Jr, Wiste HJ, Weigand SD, Rocca WA, Knopman DS, Mielke MM, et al. Age-specific population frequencies of cerebral beta-amyloidosis and neurodegeneration among people with normal cognitive function aged 5089 years: a cross-sectional study. Lancet Neurol. 2014;13(10):997-1005.

17. Reiman EM, Chen K, Liu X, Bandy D, Yu M, Lee W, et al. Fibrillar amyloidbeta burden in cognitively normal people at 3 levels of genetic risk for Alzheimer's disease. Proc Natl Acad Sci U S A. 2009;106(16):6820-5.

18. Wenham PR, Price WH, Blandell G. Apolipoprotein E genotyping by onestage PCR. Lancet. 1991;337(8750):1158-9.

19. Wirth M, Villeneuve S, Haase CM, Madison CM, Oh H, Landau SM, et al. Associations between Alzheimer disease biomarkers, neurodegeneration, and cognition in cognitively normal older people. JAMA Neurol. 2013; 70(12):1512-9.

20. Arfanakis K, Wilson RS, Barth CM, Capuano AW, Vasireddi A, Zhang S, et al. Cognitive activity, cognitive function, and brain diffusion characteristics in old age. Brain Imaging Behav. 2016;10(2):455-63.

21. Lyall DM, Cox SR, Lyall LM, Celis-Morales C, Cullen B, Mackay DF, et al. Association between APOE e4 and white matter hyperintensity volume, but not total brain volume or white matter integrity. Brain Imaging Behav. 2020; 14(5):1468-76

22. Xu G, Zheng S, Zhu Z, Yu X, Jiang J, Jiang J, et al. Association of tau accumulation and atrophy in mild cognitive impairment: a longitudinal study. Ann Nucl Med. 2020;34(11):815-23.

23. Genomes Project C, Auton A, Brooks LD, Durbin RM, Garrison EP, Kang HM, et al. A global reference for human genetic variation. Nature. 2015; 526(7571):68-74.

24. Lee S, Seo J, Park J, Nam JY, Choi A, Ignatius JS, et al. Korean Variant Archive (KOVA): a reference database of genetic variations in the Korean population. Sci Rep. 2017;7(1):4287.

25. Lek M, Karczewski KJ, Minikel EV, Samocha KE, Banks E, Fennell T, et al. Analysis of protein-coding genetic variation in 60,706 humans. Nature. 2016; 536(7616):285-91.

26. Wang J, Raskin L, Samuels DC, Shyr Y, Guo Y. Genome measures used for quality control are dependent on gene function and ancestry. Bioinformatics. 2015;31(3):318-23.

27. D'Aoust LN, Cummings AC, Laux R, Fuzzell D, Caywood L, Reinhart-Mercer L, et al. Examination of candidate exonic variants for association to Alzheimer disease in the Amish. PLoS One. 2015;10(2):e0118043.

28. Sloan CA, Chan ET, Davidson JM, Malladi VS, Strattan JS, Hitz BC, et al. ENCODE data at the ENCODE portal. Nucleic Acids Res. 2016;44(D1):D726-32.

29. Zhao PP, Yao MJ, Chang SY, Gou LT, Liu MF, Qiu ZL, et al. Novel function of PIWIL1 in neuronal polarization and migration via regulation of microtubule-associated proteins. Mol Brain. 2015;8:39.

30. Delabio R, Rasmussen L, Mizumoto I, Viani GA, Chen E, Villares J, et al. PSEN1 and PSEN2 gene expression in Alzheimer's disease brain: a new approach. J Alzheimers Dis. 2014;42(3):757-60.

31. Cacciaglia R, Molinuevo JL, Falcon C, Brugulat-Serrat A, SanchezBenavides G, Gramunt N, et al. Effects of APOE-epsilon4 allele load on brain morphology in a cohort of middle-aged healthy individuals with enriched genetic risk for Alzheimer's disease. Alzheimers Dement. 2018; 14(7):902-12

32. Kantarci K, Lowe V, Przybelski SA, Weigand SD, Senjem ML, Ivnik RJ, et al. APOE modifies the association between Abeta load and cognition in cognitively normal older adults. Neurology. 2012;78(4):232-40.
33. Liu Y, Tan L, Wang HF, Liu Y, Hao XK, Tan CC, et al. Multiple effect of APOE genotype on clinical and neuroimaging biomarkers across Alzheimer's disease spectrum. Mol Neurobiol. 2016;53(7):4539-47.

34. Liu Y, Yu JT, Wang HF, Han PR, Tan CC, Wang C, et al. APOE genotype and neuroimaging markers of Alzheimer's disease: systematic review and metaanalysis. J Neurol Neurosurg Psychiatry. 2015;86(2):127-34.

35. Mishra S, Blazey TM, Holtzman DM, Cruchaga C, Su Y, Morris JC, et al. Longitudinal brain imaging in preclinical Alzheimer disease: impact of APOE epsilon4 genotype. Brain. 2018;141(6):1828-39.

36. Apostolova LG, Risacher SL, Duran T, Stage EC, Goukasian N, West JD, et al. Associations of the top 20 Alzheimer disease risk variants with brain amyloidosis. JAMA Neurol. 2018;75(3):328-41.

37. Harrison OJ, Brasch J, Lasso G, Katsamba PS, Ahlsen G, Honig B, et al. Structural basis of adhesive binding by desmocollins and desmogleins. Proc Natl Acad Sci U S A. 2016:113(26):7160-5.

\section{Publisher's Note}

Springer Nature remains neutral with regard to jurisdictional claims in published maps and institutional affiliations.

Ready to submit your research? Choose BMC and benefit from:

- fast, convenient online submission

- thorough peer review by experienced researchers in your field

- rapid publication on acceptance

- support for research data, including large and complex data types

- gold Open Access which fosters wider collaboration and increased citations

- maximum visibility for your research: over $100 \mathrm{M}$ website views per year

At $\mathrm{BMC}$, research is always in progress.

Learn more biomedcentral.com/submissions 